Sādhanā Vol. 29, Part 5, October 2004, pp. 429-447. @ Printed in India

\title{
Deformation due to mechanical and thermal sources in generalised orthorhombic thermoelastic material
}

\author{
RAJNEESH KUMAR and LEENA RANI \\ Department of Mathematics, Kurukshetra University, Kurukshetra 136 119, India \\ e-mail: rajneesh_kuk@rediffmail.com
}

MS received 3 June 2003; revised 19 January 2004

\begin{abstract}
A dynamical two-dimensional problem of thermoelasticity has been considered to investigate the disturbance due to mechanical (horizontal or vertical) and thermal source in a homogeneous, thermally conducting orthorhombic material. Laplace-Fourier transforms are applied to basic equations to form a vector matrix differential equation, which is then solved by eigenvalue approach. The displacements, stresses and temperature distribution so obtained in the physical domain are computed numerically and illustrated graphically. The numerical results of these quantities for zinc crystal-like material are illustrated to compare the results for different theories of generalised thermoelasticity for an insulated boundary and a temperature gradient boundary.
\end{abstract}

Keywords. Thermoelastic; generalised thermoelasticity; thermal relaxation parameters; mechanical/thermal sources; Laplace and Fourier transforms.

\section{Introduction}

In many engineering phenomena, including the response of soils, geological materials and composites, the assumption of isotropic behaviour may not capture some significant features of the continuum response. The formulation and solution of anisotropic problems is far more difficult and cumbersome than its isotropic counterpart. In recent years, the elastodynamic response of anisotropic continuum has received the attention of Barnett \& Lothe (1974), Chadwick (1976), Musgrave (1981), Royer \& Dieulesaint (1984), Domanski \& Jablonski (2001), Destrade (2001).

Dhaliwal \& Sherief (1980) extended the generalised theory of thermoelasticity (Lord \& Shulman 1967) to anisotropic media. Pao and Banerjee investigated the propagation of plane harmonic waves in homogeneous anisotropic solids (Pao \& Banerjee 1973) and dielectric crystals (Banerjee \& Pao 1974) respectively, by taking thermal relaxation time into consideration. Sharma and Singh studied the propagation of thermoelastic waves in transversely isotropic material (Singh \& Sharma 1984) and cubic crystals (Sharma \& Singh 1990) respectively. Lin \& Zhao (1995) discussed plane problem of orthotropic quasi-static thermoelasticity. Sharma et al (2000) discussed plane harmonic waves in orthorhombic

A list of symbols is given at the end of the paper 
materials. Verma \& Hasebe (2001) studied wave propagation in plates of general anisotropic media in generalised thermoelasticity.

The present investigation is to determine the components of displacement, stress and temperature distribution in a homogeneous, thermally conducting orthorhombic material due to mechanical and thermal sources.

\section{Basic equations}

The basic governing equations for homogeneous, anisotropic thermoelasticity in the absence of body forces and heat sources are given by

$$
\begin{aligned}
t_{i j, j} & =\rho \ddot{u}_{i}, \\
K_{i j} T_{, i j} & =\rho c_{e}\left(\dot{T}+\tau_{o} \ddot{T}\right)+T_{o} \beta_{i j}\left(\dot{u}_{i, j}+\tau_{o} \delta_{1 k} \ddot{u}_{i, j}\right), \\
t_{i j} & =c_{i j k l} e_{k l}-\beta_{i j}\left(T+\tau_{1} \delta_{2 k} \dot{T}\right), \quad \beta_{i j}=c_{i j k l} \alpha_{k l} ;(i, j, k, l=1,2,3) .
\end{aligned}
$$

The comma notation is used for spatial derivatives and dot notation represents time differentiation, Here $k=1$ for Lord and Shulman (L-S) theory (Lord \& Shulman 1967) and $k=2$ for Green and Lindsay (G-L) theory (Green \& Lindsay 1972). Thermal relaxations $\tau_{o}$ and $\tau_{1}$ satisfy the inequality $\tau_{1} \geq \tau_{o} \geq 0$ for the $\mathrm{G}-\mathrm{L}$ theory only. $c_{i j k l}$ satisfies the (Green) symmetry conditions:

$$
c_{i j k l}=c_{k l i j}=c_{i j l k}=c_{j i k l} .
$$

Parameters in (1)-(2) are assumed to satisfy the following conditions.

(i) The thermal conductivity tensor $K_{i j}$ is symmetric and positive-definite;

(ii) The thermoelastic coupling tensor $\beta_{i j}$ is non-singular;

(iii) The specific heat $c_{e}$ at constant strain is positive;

(iv) The isothermal linear elasticities are positive-definite in the sense that

$$
c_{i j k l} e_{i j} e_{k l}>0 \text {. }
$$

\section{Formulation and solution of the problem}

We consider a homogeneous, orthorhombic thermoelastic half-space in the undeformed state at uniform temperature $T_{0}$. We take the origin on the plane surface and $z$-axis normally into the medium, which is thus represented by $z \geq 0$. A concentrated, uniformly and linearly distributed mechanical or thermal source, is assumed to be acting at the origin of the rectangular Cartesian co-ordinates.

For plane strain two-dimensional problem, we take displacement vector $\vec{u}=(u, 0, w)$ and $T(x, z, t)$ as temperature change.

Using the contracting subscript notations in equation (3) as to relate to $1 \rightarrow 11,2 \rightarrow$ $22,3 \rightarrow 33,4 \rightarrow 23,5 \rightarrow 13,6 \rightarrow 12$ to relate $c_{i j k l}$ to $c_{p q}(j, j, k, l=1,2,3$ and $p, q=$ $1,2, \ldots \ldots, 6)$.

Making use of the $c_{p q}$ from equation (3) in equations (1) and (2) then the field equations and constitutive relations for such a medium in the absence of body forces and heat sources in non-dimensional form after suppressing the primes can be rewritten as

$$
u,,_{x x}+c_{1} u,_{z z}+c_{2} w,_{x z}-\left(T+\delta_{2 k} \tau_{1} \dot{T}\right),_{x}=\ddot{u}
$$




$$
\begin{aligned}
& c_{1} w,_{x x}+c_{3} w,_{z z}+c_{2} u,,_{x z}-\bar{\beta}\left(T+\delta_{2 k} \tau_{1} \dot{T}\right),,_{z}=\ddot{w}, \\
& T,_{x x}+\bar{K} T,_{z z}=\left(\dot{T}+\tau_{o} \ddot{T}\right)+\epsilon_{1}\left\{\left(\dot{u}+\tau_{o} \delta_{1 k} \ddot{u}\right),{ }_{x}+\bar{\beta}\left(\dot{w}+\tau_{o} \delta_{1 k} \ddot{w}\right),_{z}\right\},
\end{aligned}
$$

where comma notation is used for spatial derivatives, we have defined the quantities

$$
\begin{aligned}
x^{\prime}= & \omega_{1}^{*} x / V_{1}, z^{\prime}=\omega_{1}^{*} z / V_{1}, u^{\prime}=\left(\rho V_{1} \omega_{1}^{*} / \beta_{1} T_{0}\right) u, t^{\prime}=\omega_{1}^{*} t, \\
& w^{\prime}=\left(\rho v_{1} \omega_{1}^{*} / \beta_{1} T_{0}\right) w, T^{\prime}=T / T_{0}, c_{1}=c_{55} / c_{11}, c_{2}=\left(c_{13}+c_{55}\right) / c_{11}, \\
& c_{3}=c_{33} / c_{11}, \bar{K}=K_{3} / K_{1}, \omega^{\prime}=\omega / \omega_{1}^{*}, \tau_{0}^{\prime}=\omega_{1}^{*} \tau_{0}, \tau_{1}^{\prime}=\omega_{1}^{*} \tau_{1}, \\
& a^{\prime}=\omega_{1}^{*} a / V_{1}, \bar{\beta}=\beta_{3} / \beta_{1}, \varepsilon_{1}=\beta_{1}^{2} T_{o} / \rho^{2} c_{e} V_{1}^{2}, \\
t_{z z}^{\prime}= & t_{z z} / \beta_{1} T_{0}, \quad t_{z x}^{\prime}=t_{z x} / \beta_{1} T_{0}, h^{\prime}=h V_{1} / \omega_{1}^{*},
\end{aligned}
$$

and $V_{1}=\left(c_{11} / \rho\right)^{1 / 2}$ and $\omega_{1}^{*}=c_{e} c_{11} / K_{1}$ are respectively, the velocity of compressional waves in the $x$-direction and the characteristic frequency of the medium.

The initial and regularity conditions are given by

$$
\begin{aligned}
u(x, z, 0) & =0=\dot{u}(x, z, 0), \\
w(x, z, 0) & =0=\dot{w}(x, z, 0), \\
T(x, z, 0) & =0=\dot{T}(x, z, 0) \text { for } z \geq 0,-\infty<x<\infty,
\end{aligned}
$$

and

$$
u(x, z, t)=w(x, z, t)=T(x, z, t)=0, \text { for } t>0, \text { when } z \rightarrow \infty
$$

Applying the Laplace and Fourier transforms,

$$
\hat{f}(x, z, p)=\int_{0}^{\infty} f(x, z, t) e^{-p t} \mathrm{~d} t
$$

and

$$
\tilde{f}(\xi, z, p)=\int_{-\infty}^{\infty} \hat{f}(x, z, p) e^{i \xi x} \mathrm{~d} x,
$$

on the resulting expressions, we obtain

$$
\begin{aligned}
& \tilde{u}^{\prime \prime}=R_{11} \tilde{u}+R_{13} \tilde{T}+R_{15} \tilde{w}^{\prime}, \\
& \tilde{w}^{\prime \prime}=R_{22} \tilde{w}+R_{24} \tilde{u}^{\prime}+R_{26} \tilde{T}^{\prime}, \\
& \tilde{T}^{\prime \prime}=R_{31} \tilde{u}+R_{33} \tilde{T}+R_{35} \tilde{w}^{\prime} .
\end{aligned}
$$

where primes in (12)-(14) represent the first- and second-order differentiation with respect to $z$ respectively and

$$
\begin{aligned}
R_{11}= & \left(\xi^{2}+p^{2}\right) / c_{1}, R_{13}=-i \xi\left(1+\tau_{1} p \delta_{2 k}\right) / c_{1}, R_{15}=i \xi c_{2} / c_{1}, \\
& R_{22}=\left(c_{1} \xi^{2}+p^{2}\right) / c_{3}, R_{26}=\bar{\beta}\left(1+\tau_{1} p \delta_{2 k}\right) / c_{3}, R_{24}=i \xi c_{2} / c_{3}, \\
& R_{31}=-i \xi \varepsilon_{1}\left(p+\tau_{0} p^{2} \delta_{1 k}\right) / \bar{K}, R_{33}=\left(\xi^{2}+p+\tau_{0} p^{2}\right) / \bar{K}, \\
& R_{35}=\varepsilon_{1} \bar{\beta}\left(p+\tau_{0} p^{2} \delta_{1 k}\right) / \bar{K} .
\end{aligned}
$$


Equations (12)-(14) can be written as

$$
\frac{\mathrm{d}}{\mathrm{d} z} W(\xi, z, p)=A(\xi, p) W(\xi, z, p),
$$

where

$$
\begin{aligned}
W & =\left[\begin{array}{l}
U \\
U^{\prime}
\end{array}\right], A=\left[\begin{array}{ll}
0 & I \\
\mathrm{~A}_{2} & A_{1}
\end{array}\right], U=\left[\begin{array}{l}
\tilde{u} \\
\tilde{w} \\
\tilde{T}
\end{array}\right], U^{\prime}=\left[\begin{array}{c}
\tilde{u}^{\prime} \\
\tilde{w}^{\prime} \\
\tilde{T}^{\prime}
\end{array}\right], \\
0 & =\left[\begin{array}{lll}
0 & 0 & 0 \\
0 & 0 & 0 \\
0 & 0 & 0
\end{array}\right], I=\left[\begin{array}{lll}
1 & 0 & 0 \\
0 & 1 & 0 \\
0 & 0 & 1
\end{array}\right], A_{1}=\left[\begin{array}{lll}
0 & R_{15} & 0 \\
R_{24} & 0 & R_{26} \\
0 & R_{35} & 0
\end{array}\right], \\
A_{2} & =\left[\begin{array}{lll}
R_{11} & 0 & R_{13} \\
0 & R_{22} & 0 \\
R_{31} & 0 & R_{33}
\end{array}\right] .
\end{aligned}
$$

To solve (16),we take

$$
W(\xi, z, p)=X(\xi, p) e^{q z},
$$

so that

$$
A(\xi, p) W(\xi, z, p)=q W(\xi, z, p),
$$

which leads to an eigenvalue problem. The characterstic equation corresponding to the matrix $A$ is given by

$$
\operatorname{det}[A-q I]=0,
$$

which on expansion leads to

$$
q^{6}-\lambda_{1} q^{4}+\lambda_{2} q^{2}-\lambda_{3}=0
$$

where

$$
\begin{aligned}
\lambda_{1}= & R_{15} R_{24}+R_{33}+R_{22}+R_{26} R_{35}, \\
\lambda_{2}= & R_{15} R_{24} R_{33}-R_{13} R_{24} R_{35}+R_{22} R_{33}+R_{11} R_{26} R_{35} \\
& -R_{31} R_{15} R_{26}+R_{11} R_{33}-R_{31} R_{13}+R_{11} R_{22}, \\
\lambda_{3}= & R_{22}\left(R_{11} R_{33}-R_{31} R_{13}\right) .
\end{aligned}
$$

The roots of (21) are $\pm q_{i}(i=1,2,3)$.

The eigenvalues of the matrix $A$ are the roots of (21). The eigenvector $X(\xi, p)$ corresponding to the eigenvalues $q_{i}$ can be determined by solving the homogeneous equation

$$
[A-q I] X(\xi, p)=0 .
$$


The set of eigenvectors $X_{i}(\xi, p),(i=1,2,3,4,5,6)$ may be obtained as

$$
X_{i}(\xi, p)=\left[\begin{array}{l}
X_{i 1}(\xi, p) \\
X_{i 2}(\xi, p)
\end{array}\right]
$$

where

$$
\begin{aligned}
X_{i 1}(\xi, p) & =\left[\begin{array}{c}
-\xi \\
a_{i} q_{i} \\
b_{i}
\end{array}\right], X_{i 2}(\xi, p)=\left[\begin{array}{c}
-\xi q_{i} \\
a_{i} q_{i}^{2} \\
b_{i} q_{i}
\end{array}\right], q=q_{i}, i=1,2,3 . \\
X_{j 1} & =\left[\begin{array}{c}
-\xi \\
-a_{i} q_{i} \\
b_{i}
\end{array}\right], X_{j 2}=\left[\begin{array}{c}
\xi q_{i} \\
a_{i} q_{i}^{2} \\
-b_{i} q_{i}
\end{array}\right], j=i+3, q=-q_{i}, i=1,2,3 .
\end{aligned}
$$

where

$$
\begin{aligned}
a_{i} & =\frac{-i\left\{c_{1} \bar{\beta} q_{i}^{2}+\left(c_{2}-\bar{\beta}\right) \xi^{2}-\bar{\beta} p^{2}\right\}}{\Delta_{i}}, \\
b_{i} & =\frac{-i\left(\xi^{2}+p^{2}-c_{1} q_{i}^{2}\right)\left(c_{1} \xi^{2}+p^{2}-c_{3} q_{i}^{2}\right)+q_{i}^{2} c_{2}^{2} \xi^{2}}{\left(1+\tau_{1} p \delta_{2 k}\right) \Delta_{i}} \\
\Delta_{i} & =\left\{\left(c_{3}-c_{2} \bar{\beta}\right) q_{i}^{2}-c_{1} \xi^{2}-p^{2}\right\}, \quad i=1,2,3 .
\end{aligned}
$$

The solution of (16) is given by

$$
W(\xi, z, p)=\sum_{i=1}^{3}\left[B_{i} X_{i}(\xi, p) \exp \left(q_{i} z\right)+B_{i+3} X_{i+3}(\xi, p) \exp \left(-q_{i} z\right)\right],
$$

where $B_{i}(i=1,2,3,4,5,6)$ are arbitrary constants.

Thus (23) represents the solution of the general problem in the plane strain case of generalised homogeneous thermoelasticity on employing the eigenvalue approach and therefore can be applied to a broad class of problems in the Laplace and Fourier transforms. Displacements and temperature distribution that satisfy the regularity conditions (10) are given by

$$
\begin{aligned}
& \tilde{u}(\xi, z, p)=-\xi\left(B_{4} e^{-q_{1} z}+B_{5} e^{-q_{2} z}+B_{6} e^{-q_{3} z}\right), \\
& \tilde{w}(\xi, z, p)=-\left(a_{1} q_{1} B_{4} e^{-q_{1} z}+a_{2} q_{2} B_{5} e^{-q_{2} z}+a_{3} q_{3} B_{6} e_{3}^{-q z}\right), \\
& \tilde{T}(\xi, z, p)=\left(b_{1} B_{4} e^{-q_{1} z}+b_{2} B_{5} e^{-q_{2} z}+b_{3} B_{6} e^{-q_{3} z}\right),
\end{aligned}
$$

\section{Applications}

\subsection{Impulsive line tractions on the surface of half-space}

The boundary conditions in this case are

$$
\begin{aligned}
t_{z z}(x, z, t)= & -\psi(x) \delta(t), t_{z x}(x, z, t)=-\zeta(x) \delta(t), \\
& (\partial T / \partial z)+h T=0, \text { at } z=0,
\end{aligned}
$$


where $\delta(t)$ is Dirac's delta function and $\psi(x), \zeta(x)$ specify the vertical and horizontal traction distribution functions respectively along the $x$-axis, $h$ is heat transfer coefficient.

Applying the Laplace and Fourier transforms defined by (11), we get

$$
\tilde{t}_{z z}(\xi, z, p)=-\tilde{\psi}(\xi), \tilde{t}_{z x}(\xi, z, p)=-\tilde{\zeta}(\xi),(\mathrm{d} \tilde{T} / \mathrm{d} z)+h \tilde{T}=0 .
$$

Using (7)-(9), applying the transforms defined by (11) and with the help (24)-(26) in the boundary conditions (28), we obtain the expressions for displacement components, stresses and temperature distribution as

$$
\begin{aligned}
\tilde{u}= & (\xi / \Delta)\left\{\tilde{\psi}(\xi)\left(\Delta_{1}^{\prime} \bar{e}^{q_{1} z}-\Delta_{3}^{\prime} \bar{e}^{q_{2} z}+\Delta_{5}^{\prime} \bar{e}^{q_{3} z}\right)\right. \\
& \left.-\tilde{\zeta}(\xi)\left(\Delta_{2}^{\prime} \bar{e}^{q_{1} z}-\Delta_{4}^{\prime} \bar{e}^{q_{2} z}+\Delta_{6}^{\prime} \bar{e}^{q_{3} z}\right)\right\}, \\
\tilde{w}= & (1 / \Delta)\left\{\tilde{\psi}(\xi)\left(a_{1} q_{1} \Delta_{1}^{\prime} \bar{e}^{q_{1} z}-a_{2} q_{2} \Delta_{3}^{\prime} \bar{e}^{q_{2} z}+a_{3} q_{3} \Delta_{5}^{\prime} \bar{e}^{q_{3} z}\right)\right. \\
& \left.-\tilde{\zeta}(\xi)\left(a_{1} q_{1} \Delta_{2}^{\prime} \bar{e}^{q_{1} z}-a_{2} q_{2} \Delta_{4}^{\prime} \bar{e}^{q_{2} z}+a_{3} q_{3} \Delta_{6}^{\prime} \bar{e}^{q_{3} z}\right)\right\}, \\
\tilde{T}= & (-1 / \Delta)\left\{\tilde{\psi}(\xi)\left(b_{1} \Delta_{1}^{\prime} \bar{e}^{q_{1} z}-b_{2} \Delta_{3}^{\prime} \bar{e}^{q_{2} z}+b_{3} \Delta_{5}^{\prime} \bar{e}^{q_{3} z}\right)\right. \\
& \left.-\tilde{\zeta}(\xi)\left(b_{1} \Delta_{2}^{\prime} \bar{e}^{q_{1} z}-b_{2} \Delta_{4}^{\prime} \bar{e}^{q_{2} z}+b_{3} \Delta_{6}^{\prime} \bar{e}^{q_{3} z}\right)\right\}, \\
\tilde{t}_{z z}= & (-1 / \Delta)\left\{\tilde{\psi}(\xi)\left(p_{1} \Delta_{1}^{\prime} \bar{e}^{q_{1} z}-p_{2} \Delta_{3}^{\prime} \bar{e}^{q_{2} z}+p_{3} \Delta_{5}^{\prime} \bar{e}^{q_{3} z}\right)\right. \\
& \left.-\tilde{\zeta}(\xi)\left(p_{1} \Delta_{2}^{\prime} \bar{e}^{q_{1} z}-p_{2} \Delta_{4}^{\prime} \bar{e}^{q_{2} z}+p_{3} \Delta_{6}^{\prime} \bar{e}^{q_{3} z}\right)\right\}, \\
\tilde{t}_{z x}= & (-1 / \Delta)\left\{\tilde{\psi}(\xi)\left(m_{1} \Delta_{1}^{\prime} \bar{e}^{q_{1} z}-m_{2} \Delta_{3}^{\prime} \bar{e}^{q_{2} z}+m_{3} \Delta_{5}^{\prime} \bar{e}^{q_{3} z}\right)\right. \\
& \left.-\tilde{\zeta}(\xi)\left(m_{1} \Delta_{2}^{\prime} \bar{e}^{q_{1} z}-m_{2} \Delta_{4}^{\prime} \bar{e}^{q_{2} z}+m_{3} \Delta_{6}^{\prime} \bar{e}^{q_{3} z}\right)\right\},
\end{aligned}
$$

where

$$
\begin{aligned}
\Delta & =\Delta_{1}^{*}+h \Delta_{2}^{*}, \\
\Delta_{1}^{*} & =p_{1}\left(m_{3} q_{2} b_{2}-m_{2} q_{3} b_{3}\right)+p_{2}\left(m_{3} q_{3} b_{3}-m_{3} q_{1} b_{1}\right)+p_{3}\left(m_{2} q_{2} b_{1}-m_{1} q_{2} b_{2}\right), \\
\Delta_{2}^{*} & =p_{1}\left(m_{2} b_{3}-m_{3} b_{2}\right)+p_{2}\left(m_{3} b_{1}-m_{1} b_{3}\right)+p_{3}\left(m_{1} b_{2}-m_{2} b_{1}\right), \\
\Delta_{1}^{\prime} & =\left(m_{3} q_{2}-m_{2} q_{3}\right)+h\left(m_{2}-m_{3}\right), \\
\Delta_{2}^{\prime} & =\left(p_{3} q_{2}-p_{2} q_{3}\right)+h\left(p_{2}-p_{3}\right), \\
\Delta_{3}^{\prime} & =\left(m_{3} q_{1}-m_{1} q_{3}\right)+h\left(m_{1}-m_{3}\right), \\
\Delta_{4}^{\prime} & =\left(p_{3} q_{1}-p_{1} q_{3}\right)+h\left(p_{1}-p_{3}\right), \\
\Delta_{5}^{\prime} & =\left(m_{2} q_{1}-m_{1} q_{2}\right)+h\left(m_{1}-m_{2}\right), \\
\Delta_{6}^{\prime} & =\left(p_{2} q_{1}-p_{1} q_{2}\right)+h\left(p_{1}-p_{2}\right), \\
m_{i} & =\xi k_{i}\left(1+i a_{i}\right) / c_{11}, \\
p_{i} & =\left\{i \xi c_{13}+a_{i} q_{i}^{2} c_{33}-\bar{\beta} b_{i}\left(1+p \tau_{1} \delta_{2 k}\right)\right\} / c_{11}, i=1,2,3 .
\end{aligned}
$$

Case (1a)-Concentrated line force: In this case

$$
\zeta(x)=P \delta(x), \psi(x)=P \delta(x),
$$

with

$$
\tilde{\zeta}(\xi)=P, \tilde{\psi}(\xi)=P,
$$

where $P$ is the magnitude of force. 
Case $(1 b)$-Uniform pressure: The solution due to the distributed force applied on the halfspace surface is obtained by setting

$$
\{\zeta(x), \psi(x)\}=\left\{\begin{array}{lll}
1 & \text { if } & |x| \leqslant a, \\
0 & \text { if } & |x|>a,
\end{array}\right.
$$

in (27). The Laplace and Fourier transforms with respect to the pair $(x, \xi)$ for the case of a uniform strip load of unit amplitude and width 2 a applied at the origin of the coordinate system $(x=z=0)$ in dimensionless form after suppressing the primes becomes

$$
\{\tilde{\zeta}(\xi), \tilde{\psi}(\xi)\}=\left[2 \sin \left(\xi c_{2} a / \omega_{1}^{*}\right) / \xi\right] \xi \neq 0 .
$$

Case (1c)-Linearly distributed force: The solution due to linearly distributed force applied on the half-space surface is obtained by setting

$$
\{\zeta(x), \psi(x)\}=\left\{\begin{array}{lll}
1-(|x| / a) & \text { if } & |x| \leqslant a, \\
0 & \text { if } & |x|>a,
\end{array}\right.
$$

in relations (27) where $2 a$ is the width of the strip load. Using (7)-(8) and applying the transforms defined by (11), we get

$$
\{\tilde{\zeta}(\xi), \tilde{\psi}(\xi)\}=2\left[\left(1-\cos \left(\xi c_{2} a / \omega_{1}^{*}\right)\right)\right] /\left(\xi^{2} c_{2} a / \omega_{1}^{*}\right) .
$$

The expressions for displacements, stresses and temperature distribution can be obtained for a concentrated, uniformly and linearly distributed force by replacing $\tilde{\zeta}(\xi), \tilde{\psi}(\xi)$ from (30)(32) respectively, in (29).

\subsection{Thermoelastic interactions due to thermal sources}

The boundary conditions in this case are

$$
\begin{aligned}
& t_{z z}=0 \quad t_{z x}=0, \quad \text { at } \quad z=0, \\
& \frac{\partial T}{\partial z}(x, z=0)=r(x, t) \text { at } z=0, \text { for the temperature gradient boundary, }
\end{aligned}
$$

or

$$
T(x, z=0)=r(x, t) \text { at } z=0 \text {, for the temperature input boundary. }
$$

where $r(x, t)=\tilde{\tau}_{1}(x) \delta(t)$.

Applying the Laplace and Fourier transforms defined by (11), we get

$$
r(\xi, p)=\tilde{\tau}_{1}(\xi) .
$$

Using equations (7)-(9), applying the transforms defined by (11) and with the help of equations (24)-(26) in the boundary conditions (34), we obtain the expressions for displacement components, stresses, temperature distribution as

$$
\tilde{u}=\left(-\xi \tilde{\tau}_{1}(\xi) / \Delta\right)\left(\Delta_{1}^{\prime \prime} \bar{e}^{q_{1} z}-\Delta_{2}^{\prime \prime} \bar{e}^{q_{2} z}+\Delta_{3}^{\prime \prime} \bar{e}^{q_{3} z}\right),
$$




$$
\begin{aligned}
\tilde{w} & =\left(-\tilde{\tau}_{1}(\xi) / \Delta\right)\left(a_{1} q_{1} \Delta_{1}^{\prime \prime} \bar{e}^{q_{1} z}-a_{2} q_{2} \Delta_{2}^{\prime \prime} \bar{e}^{q_{2} z}+a_{3} q_{3} \Delta_{3}^{\prime \prime} \bar{e}^{q_{3} z}\right) \\
\tilde{T} & =\left(-\tilde{\tau}_{1}(\xi) / \Delta\right)\left(b_{1} \Delta_{1}^{\prime \prime} \bar{e}^{q_{1} z}-b_{2} \Delta_{2}^{\prime \prime} \bar{e}^{q_{2} z}+b_{3} \Delta_{3}^{\prime \prime} \bar{e}^{q_{3} z}\right) \\
\tilde{t}_{z z} & =\left(\tilde{\tau}_{1}(\xi) / \Delta\right)\left(p_{1} \Delta_{1}^{\prime \prime} \bar{e}^{q_{1} z}-p_{2} \Delta_{2}^{\prime \prime} \bar{e}^{q_{2} z}+p_{3} \Delta_{3}^{\prime \prime} e^{q_{3} z}\right) \\
\tilde{t}_{z x} & =\left(\tilde{\tau}_{1}(\xi) / \Delta\right)\left(m_{1} \Delta_{1}^{\prime \prime} e^{q_{1} z}-m_{2} \Delta_{2}^{\prime \prime} e^{q_{2} z}+m_{3} \Delta_{3}^{\prime \prime} e^{q_{3} z}\right)
\end{aligned}
$$

where

$$
\begin{aligned}
\Delta_{1}^{\prime \prime} & =p_{2} m_{3}-m_{2} p_{3}, \\
\Delta_{2}^{\prime \prime} & =p_{2} m_{3}-m_{1} p_{3}, \\
\Delta_{3}^{\prime \prime} & =p_{1} m_{2}-m_{1} p_{2} .
\end{aligned}
$$

On replacing $\Delta$ by $\Delta_{1}^{*}$ and $\Delta_{2}^{*}$ respectively, we obtain the expressions for temperature gradient boundary and temperature input boundary.

Case (2a)-Thermal source: In this case

$$
\tilde{\tau}_{1}(x)=P \delta(x),
$$

with

$$
\tilde{\tau}_{1}(\xi)=P,
$$

where $P$ is the magnitude of constant temperature applied on the boundary.

Case (2b) - Uniformly distributed thermal source: In this case

$$
\tilde{\tau}_{1}(x)=\left\{\begin{array}{lll}
1 & \text { if } & |x| \leqslant a, \\
0 & \text { if } & |x|>a,
\end{array}\right.
$$

with

$$
\tilde{\tau}_{1}(\xi)=2 \sin \left(\xi c_{2} a / \omega_{1}^{*}\right) / \xi, \xi \neq 0 .
$$

Case (2c) - Linearly distributed thermal source: In this case

$$
\tilde{\tau}_{1}(x)=\left\{\begin{array}{lll}
1-(|x| / a) & \text { if } & |x| \leqslant a, \\
0 & \text { if } & |x|>a,
\end{array}\right.
$$

with

$$
\tilde{\tau}_{1}(\xi)=2\left[\left(1-\cos \left(\xi c_{2} a / \omega_{1}^{*}\right)\right)\right] /\left(\xi^{2} c_{2} a / \omega_{1}^{*}\right) .
$$

The expressions for displacements, stresses and temperature distribution can be obtained for a thermal point source, and uniformly and linearly distributed thermal sources by replacing $\tilde{\tau}_{1}(\xi)$ from (36)-(38) respectively, in (35).

Sub-case 1: If $h \rightarrow 0,(29)$ yields the expressions for displacements, stresses and temperature distribution for the insulated boundary. 
Sub-case 2: If $h \rightarrow \infty$, (29) yields the expressions of displacements, stresses, and temperature distribution for the isothermal boundary.

Special case 1: By putting $k=1$ and $\tau_{1}=0$ in equations (29) and (35), we obtain the displacements, stresses and temperature distribution for L-S theory (Lord \& Shulman 1967).

Special case 2: For G-L theory (Green \& Lindsay 1972), we obtain the corresponding expressions for displacements, stresses and temperature distribution by substituting $k=2$ in (29) and (35).

\section{Inversion of the transforms}

To obtain the solutions of the problems in the physical domain, we must invert the transforms in (29) and (35), for the two theories, i.e., L-S (Lord \& Shulman 1967) and G-L (Green \& Lindsay 1972). These expressions are functions of $z$, the parameters of Laplace and Fourier transforms $p$ and $\xi$ respectively, and hence are of the form $\tilde{\mathbf{f}}(\xi, z, p)$. To get the function $f(x, z, t)$ in the physical domain, first we invert the Fourier transform using

$$
\hat{f}(x, z, p)=\frac{1}{2 \pi} \int_{-\infty}^{\infty} e^{-i \xi x} \tilde{f}(\xi, z, p) \mathrm{d} \xi=\frac{1}{\pi} \int_{0}^{\infty}\left(\cos (\xi x) f_{e}-i \sin (\xi x) f_{0}\right) \mathrm{d} \xi,
$$

where $f_{e}$ and $f_{0}$ are respectively the even and odd parts of the function $\tilde{\mathbf{f}}(\xi, z, p)$. Thus, expression (39) gives us the Laplace transform $\hat{\mathbf{f}}(x, z, p)$ of the function $f(x, z, t)$.

Then, for the fixed values of $\xi, x$ and $z$ the $\hat{\mathbf{f}}(x, z, p)$ in the expression (39) can be considered as the Laplace transforms $\hat{\mathbf{g}}(\mathbf{p})$ of $\mathrm{g}(\mathrm{t})$. Following Honig \& Hirdes (1984), the Laplace transformed function $\hat{\mathbf{g}}(\mathbf{p})$ can be inverted as given below. The function $g(t)$ can be obtained by using

$$
g(t)=\frac{1}{2 \pi i} \int_{C-i \infty}^{C+i \infty} e^{p t} \hat{g}(p) \mathrm{d} p
$$

where $C$ is an arbitrary real number greater than all the real parts of the singularities of $\hat{g}(p)$. Taking $p=C+i y$ we get

$$
g(t)=\frac{e^{C t}}{2 \pi} \int_{-\infty}^{\infty} e^{i t y} \hat{g}(C+i y) \mathrm{d} y .
$$

Now, taking $e^{-C t} g(t)$ as $h(t)$ and expanding it as Fourier series in $[0,2 L]$, we obtain approximately the formula

$$
g(t)=g_{\infty}(t)+E_{D},
$$

where

$$
g_{\infty}(t)=\frac{C_{0}}{2}+\sum_{k=1}^{\infty} C_{k}, \quad 0 \leqslant t \leqslant 2 L,
$$


and

$$
C_{k}=\left(e^{C t} / L\right) \operatorname{Re}\left[e^{i k \pi t / L} \hat{g}(C+(i k \pi / L))\right] .
$$

$E_{D}$ is the discretization error and can be made arbitrarily small by choosing $C$ large enough. The value of $C$ and $L$ are chosen according to the criteria outlined by Honig \& Hirdes (1984).

Since the infinite series in (42) can be summed up only to a finite number of $N$ terms, so the approximate value of $g(t)$ becomes

$$
g_{N}(t)=\frac{C_{0}}{2}+\sum_{k=1}^{N} C_{k}, \quad 0 \leqslant t \leqslant 2 L,
$$

Now, we introduce a truncation error $E_{T}$, that must be added to the discretization error to produce the total approximate error in evaluating $g(t)$ using the above formula. To accelerate the convergence, the discretization error and then the truncation error is reduced by using the 'Korrecktur method' and the ' $\in$-algorithm', respectively as given by Honig \& Hirdes (1984).

The Korrecktur method formula, to evaluate the function $g(t)$ is

$$
g(t)=g_{\infty}(t)-e^{-2 C L} g_{\infty}(2 L+t)+E_{D}^{\prime},
$$

where

$$
\left|E_{D}^{\prime}\right| \ll\left|E_{D}\right|
$$

Thus, the approximate value of $g(t)$ becomes

$$
g_{N_{K}}(t)=g_{N}(t)-e^{-2 C L} g_{N^{\prime}}(2 L+t),
$$

where $N^{\prime}$ is an integer such that $N^{\prime}<N$.

We shall now describe the $\in$-algorithm, which is used to accelerate the convergence of the series in (43). Let $N$ be an odd natural number and $S_{m}=\sum_{k=1}^{m} C_{k}$ be the sequence of partial sums of (43). We define the ' $\in$-sequence' by

$$
\epsilon_{0, m}=0, \epsilon_{1, m}=S_{m}, \epsilon_{n+1, m}=\epsilon_{n-1, m+1}+\frac{1}{\epsilon_{n, m+1}-\epsilon_{n, m}} ; n, m=1,2,3, \ldots \ldots
$$

The sequence $\epsilon_{1,1}, \epsilon_{3,1}, \ldots \ldots, \in_{N, 1}$ converges to $g(t)+E_{D}-\left(C_{0} / 2\right)$ faster than the sequence of partial sums $S_{m}, m=1,2,3, \ldots$. The actual procedure to invert the Laplace transform consists of (44) together with the ' $\in$-algorithm'.

The last step is to calculate the integral in (39). The method for evaluating this integral is described by Press et al (1986), and involves the use of Romberg's integration with adaptive step size. This also uses the results from successive refinements of the extended trapezoidal rule followed by extrapolation of the results to the limit when the step size tends to zero.

\section{Numerical result and discussion}

The material chosen for the purpose of numerical evaluation was zinc which falls in the category of transversely isotropic materials. Physical data for a single crystal of zinc given by Dhaliwal \& Singh (1980) are as below. 


$$
\begin{aligned}
\varepsilon= & 0.0221, c_{1}=0.4729, c_{2}=0.7850, \rho=7.14 \times 10^{3} \mathrm{kgm}^{-3}, c_{3}=0.3851, \\
& T_{0}=296 \mathrm{~K}, c_{e}=3.9 \times 10^{2} \mathrm{Jkg}^{-1} \text { degree }^{-1}, \bar{\beta}=0.8991, \\
& \omega_{1}^{*}=5.01 \times 10^{11} \mathrm{~s}^{-1}, \bar{K}=1 \cdot 0, P=1 .
\end{aligned}
$$

The variations of normal stress $t_{z z}$ and boundary temperature field $T$ with distance $x$ are shown graphically in figures 1-12, for L-S (Lord \& Shulman 1967) and G-L (Green \& Lindsay 1972) theories for non-dimensional relaxation times $\tau_{0}=0 \cdot 02, \tau_{1}=0 \cdot 05$. At $t=0 \cdot 5$, L-S (Lord \& Shulman 1967) and G-L (Green \& Lindsay 1972) are shown by solid line (- - ), and dashed line (- - ) respectively, and for $t=1, \mathrm{~L}-\mathrm{S}$ (Lord \& Shulman 1967) and G-L (Green $\&$ Lindsay 1972) are shown by solid line, and dashed line with circles (०-० - ) respectively. Results for distributed load and distributed thermal point source are for dimensionless width, $a=1$.

\subsection{Mechanical force on the boundary of half-space (insulated boundary)}

6.1a Concentrated force: Figure 1 shows the variation of normal stress $t_{z z}$ with distance $x$. At the point of application of source, the values of normal stress $t_{z z}$ at time $t=1.0$ are greater than those at time $t=0.5$ for both the theories. The value of $t_{z z}$ remain maximum at time $t=1.0$ in the whole range.

Figure 2 shows the variation of temperature distribution $T$ with distance $x$. Initially the values of temperature distribution at $t=1.0$ are less than those at time $t=0.5$ for both the theories. At time $t=0.5$ the values of temperature distribution for G-L (Green \& Lindsay 1972) theory are greater than that of the L-S (Lord \& Shulman 1967) theory in the whole range whereas at $t=1.0$ the values of temperature distribution for L-S (Lord \& Shulman

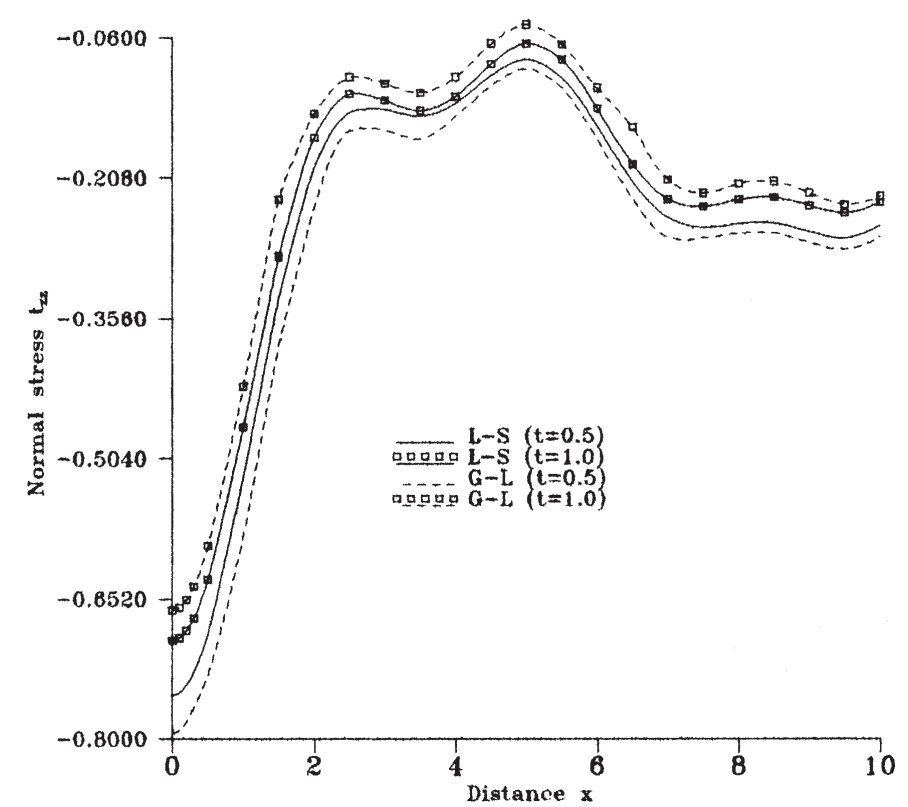

Figure 1. Variation of normal stress $t_{z z}$ with distance $x$ due to concentrated force along normal direction (insulated boundary). 


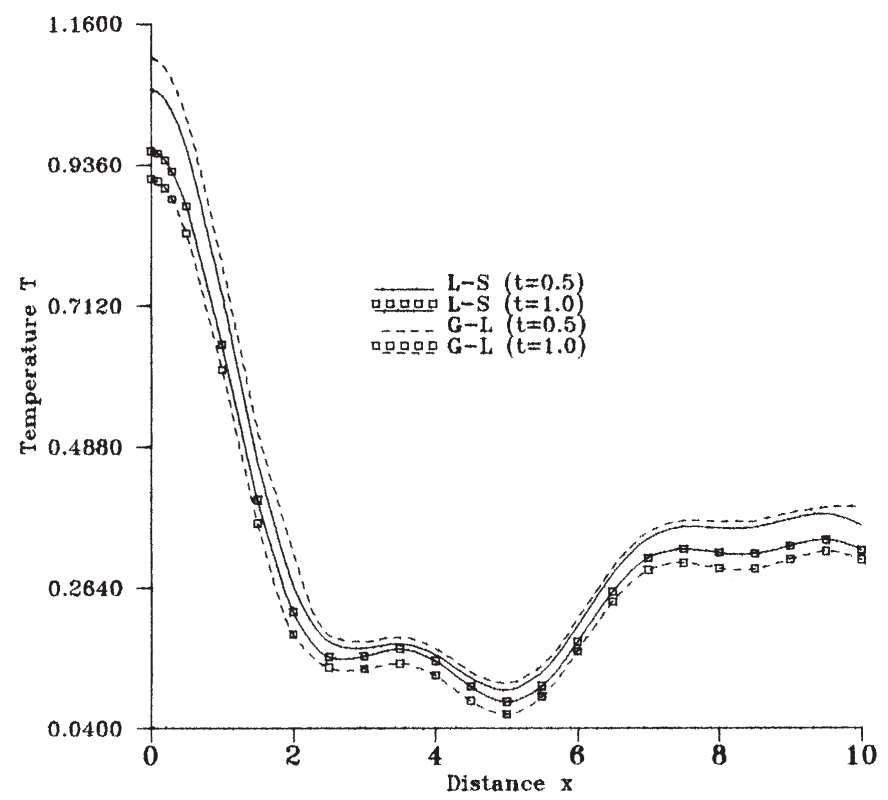

Figure 2. Variation of temperature $T$ with distance $x$ due to concentrated force along the normal direction (insulated boundary).

1967) theory are greater than that of the G-L (Green \& Lindsay 1972) theory in the range $0 \leqslant x \leqslant 10$. The behaviour of variation is oscillatory in the whole range for both theories.

6.1b Uniformly and linearly distributed load: Variations of normal stress and temperature distribution for uniformly and linearly distributed forces are the same as those for concentrated force with difference in magnitudes for both the theories and for both times. These variations are shown in figures 3-6 respectively.

\subsection{Thermal sources on the surface of half-space (temperature gradient boundary)}

6.2a Thermal point source: Figure 7 shows the variation of normal stress $t_{z z}$ with distance $x$. At the point of application of source the values of normal stress $t_{z z}$ for G-L theory (Green \& Lindsay 1972) are greater than for the L-S theory (Lord \& Shulman 1967) for both times. For (Lord \& Shulman 1967) L-S theory at time $t=1.0$ the values of $t_{z z}$ are greater than those at time $t=0.5$ in the ranges $0 \leqslant x \leqslant 2,4.5 \leqslant x \leqslant 6.2$ and $8 \leqslant x \leqslant 10$ and reveals a reverse pattern in the rest of the ranges. For the (Green \& Lindsay 1972) G-L theory a similar trend of variation is observed.

Figure 8 shows the variation of temperature distribution $T$ with distance $x$. The values of temperature distribution decrease sharply and then become oscillatory in the range $2 \leqslant x \leqslant 10$ for both the theories and for both times.

6.2b Uniformly and linearly distributed thermal sources: Variations of normal stress and temperature distribution for uniformly and linearly distributed thermal sources are the same as those of thermal point sources with difference in their magnitudes for both the theories as time increases from $t=0.5$ to $t=1.0$. These variations are shown in figures 9-12 respectively. 


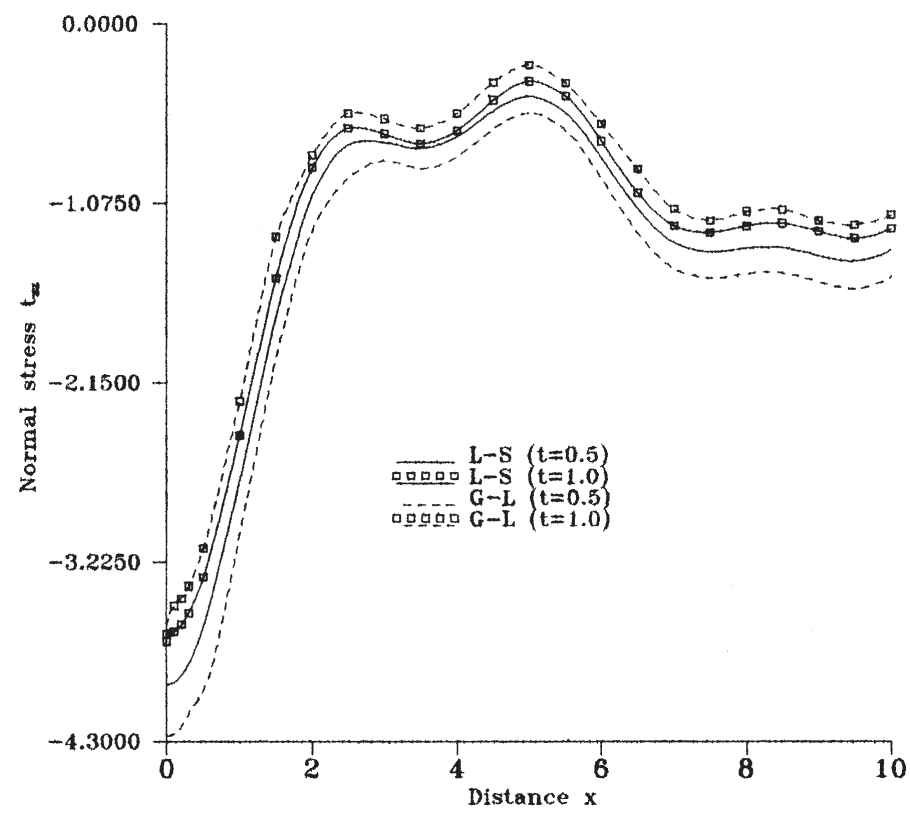

Figure 3. Variation of normal stress $t_{z z}$ with distance $x$ due to uniformly distributed force along normal direction (insulated boundary).

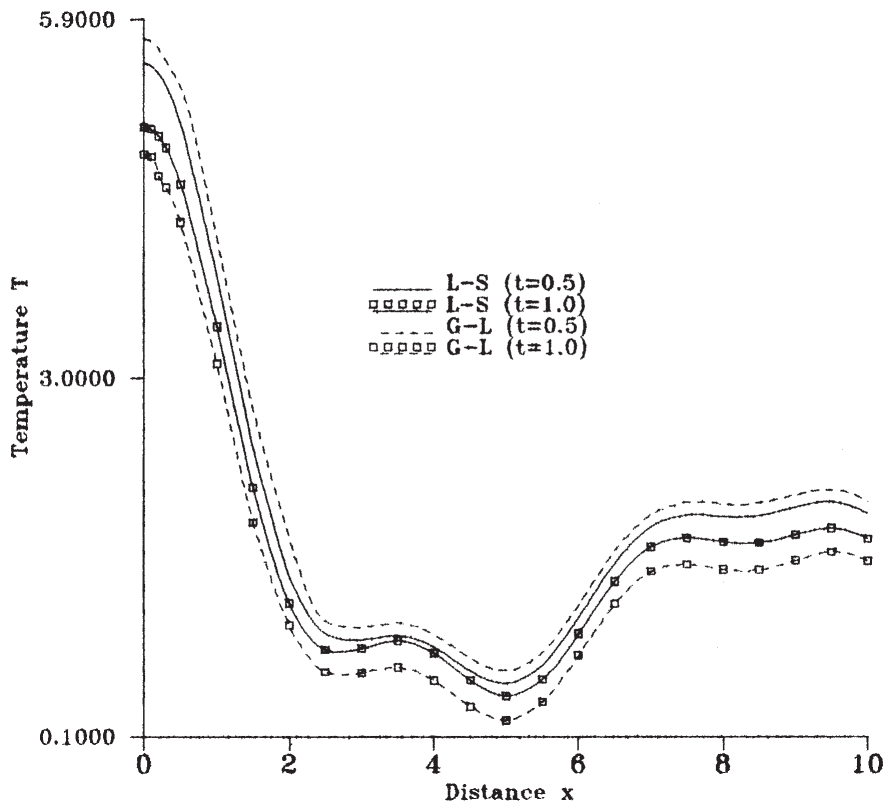

Figure 4. Variation of temperature $T$ with distance $x$ due to uniformly distributed force along the normal direction (insulated boundary). 


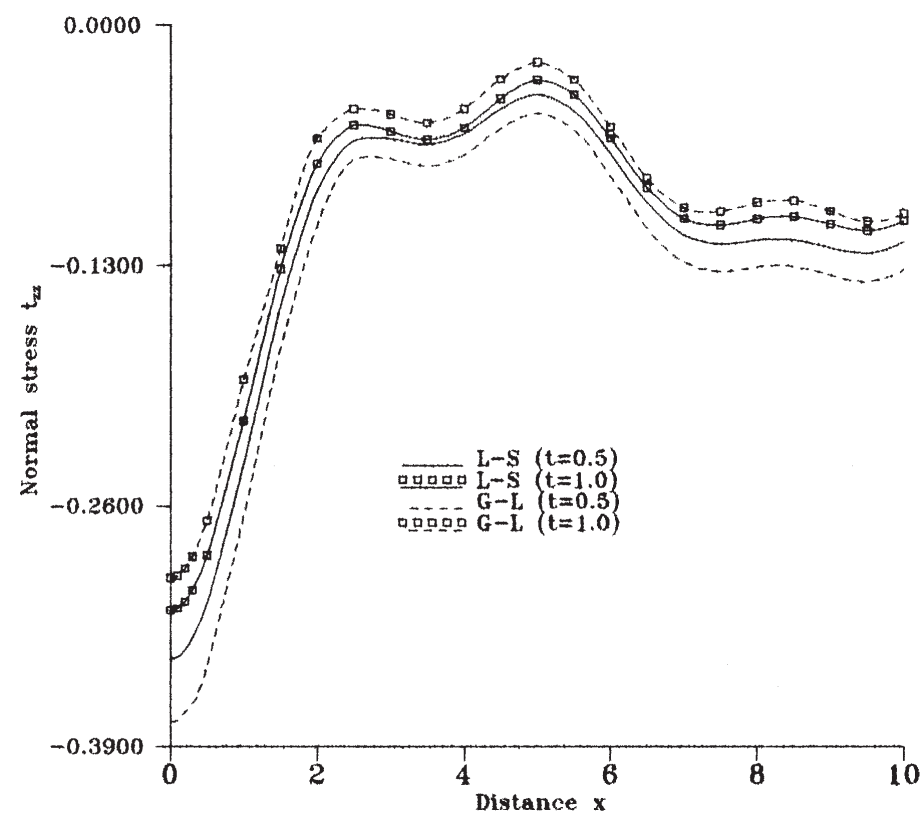

Figure 5. Variation of normal stress $t_{z z}$ with distance $x$ due to linearly distributed force along normal direction (insulated boundary).

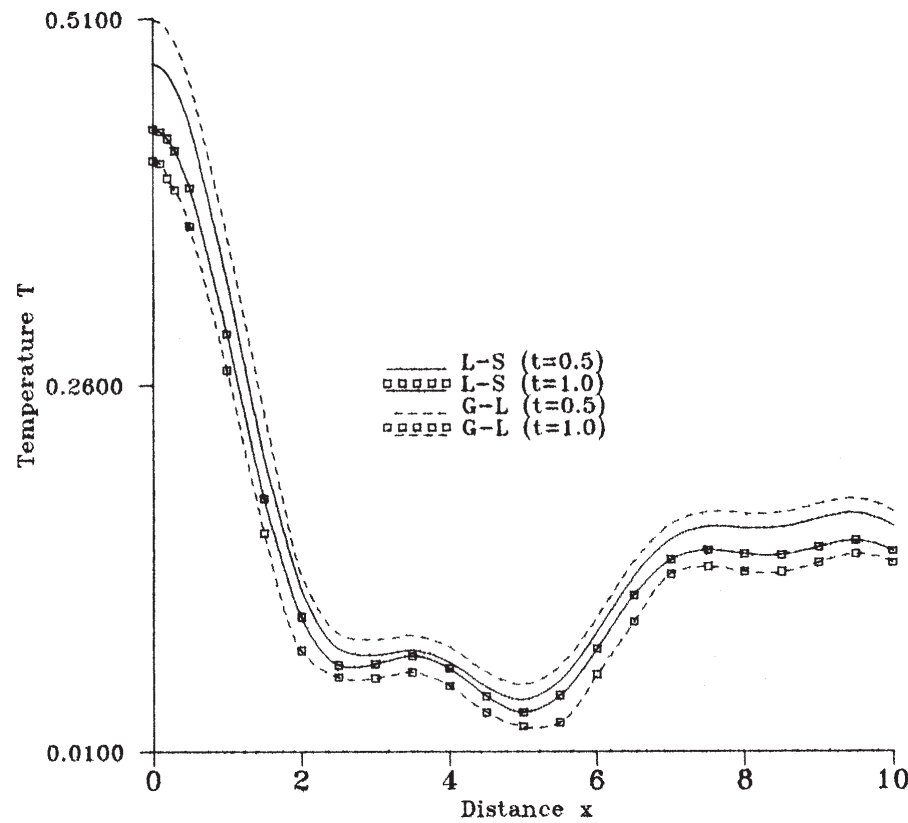

Figure 6. Variation of temperature $T$ with distance $x$ due to linearly distributed force along normal direction (insulated boundary). 


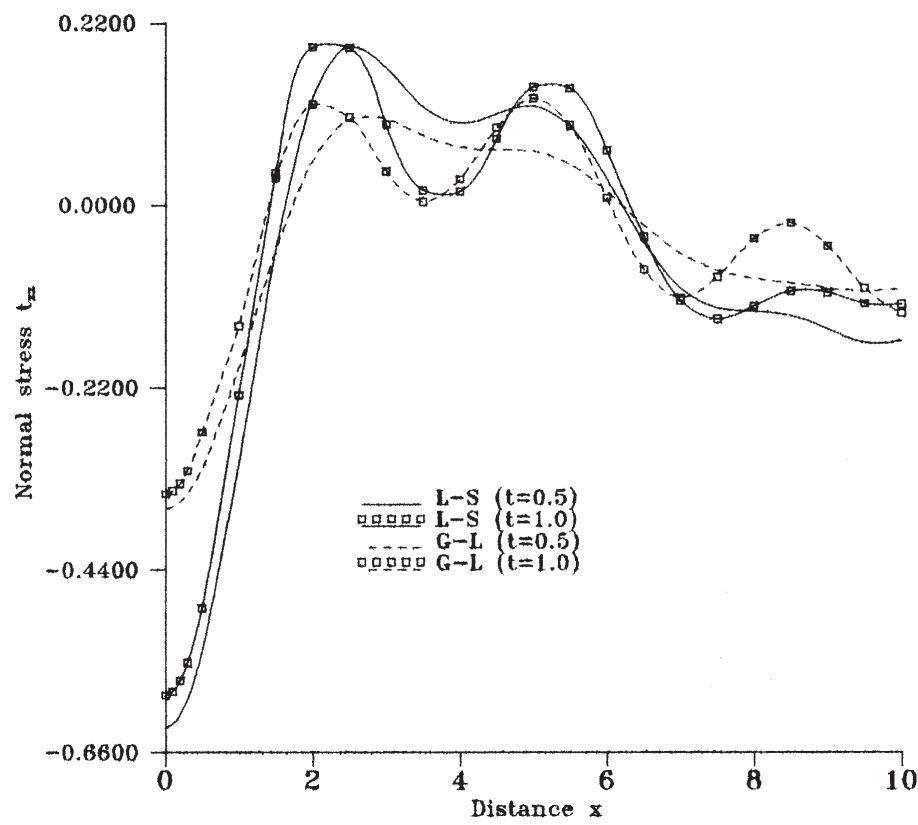

Figure 7. Variation of normal stress $t_{z z}$ with distance $x$ due to thermal point source (temperature gradient boundary).

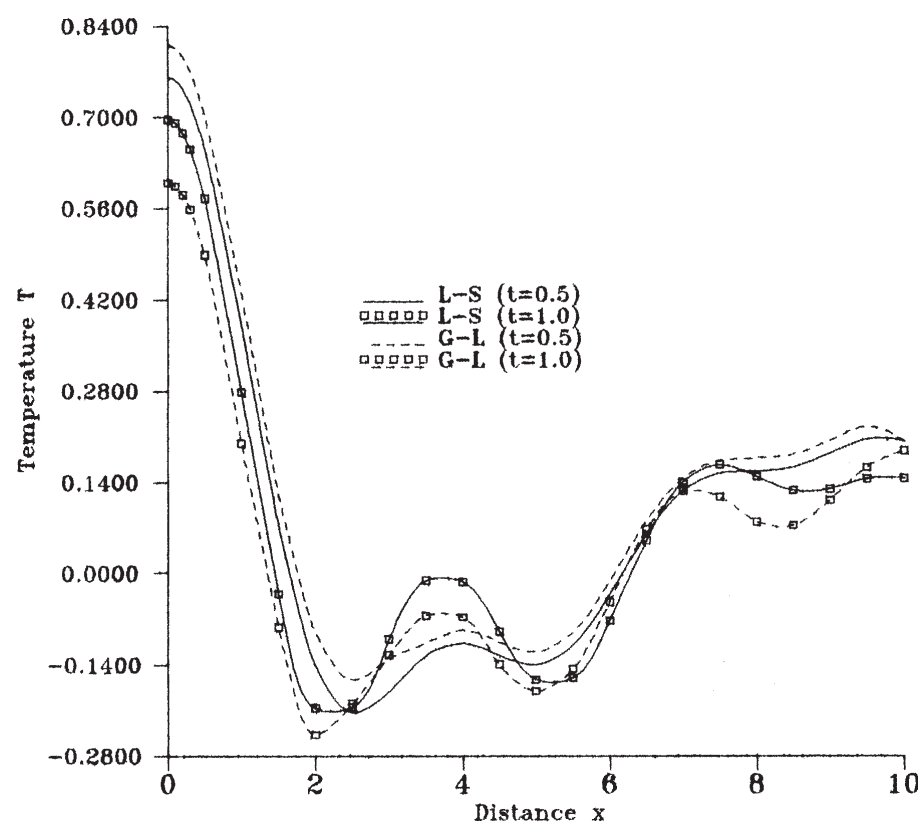

Figure 8. Variation of temperature $T$ with distance $x$ due to thermal point source (temperature gradient boundary). 


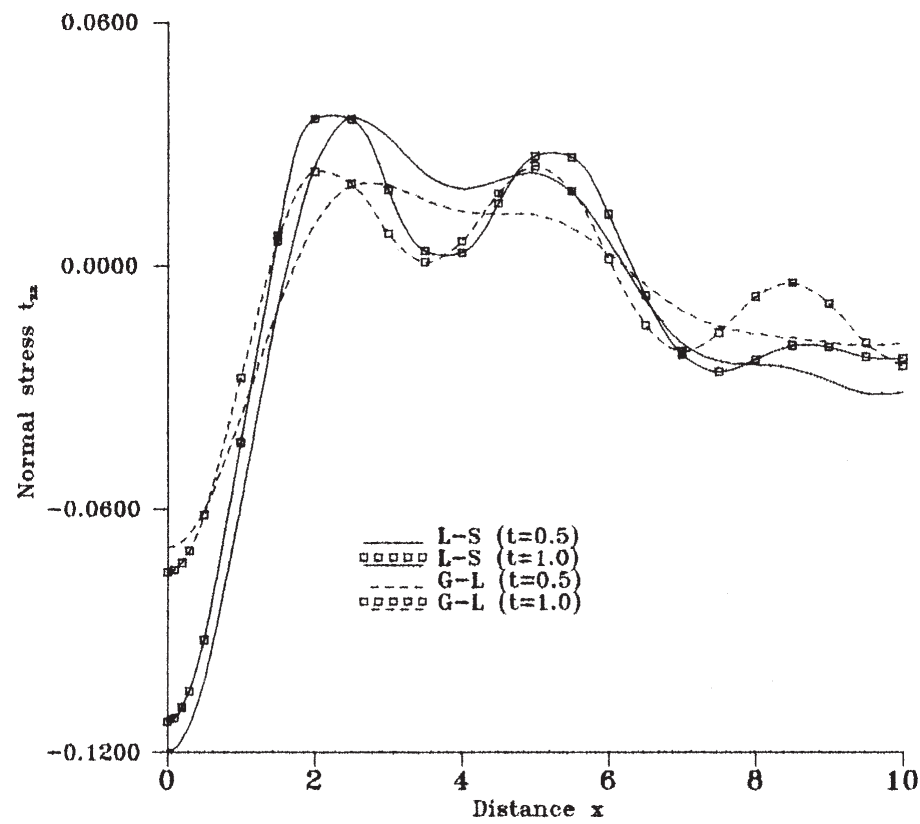

Figure 9. Variation of normal stress $t_{z z}$ with distance $x$ due to uniformly distributed thermal source (temperature gradient boundary).

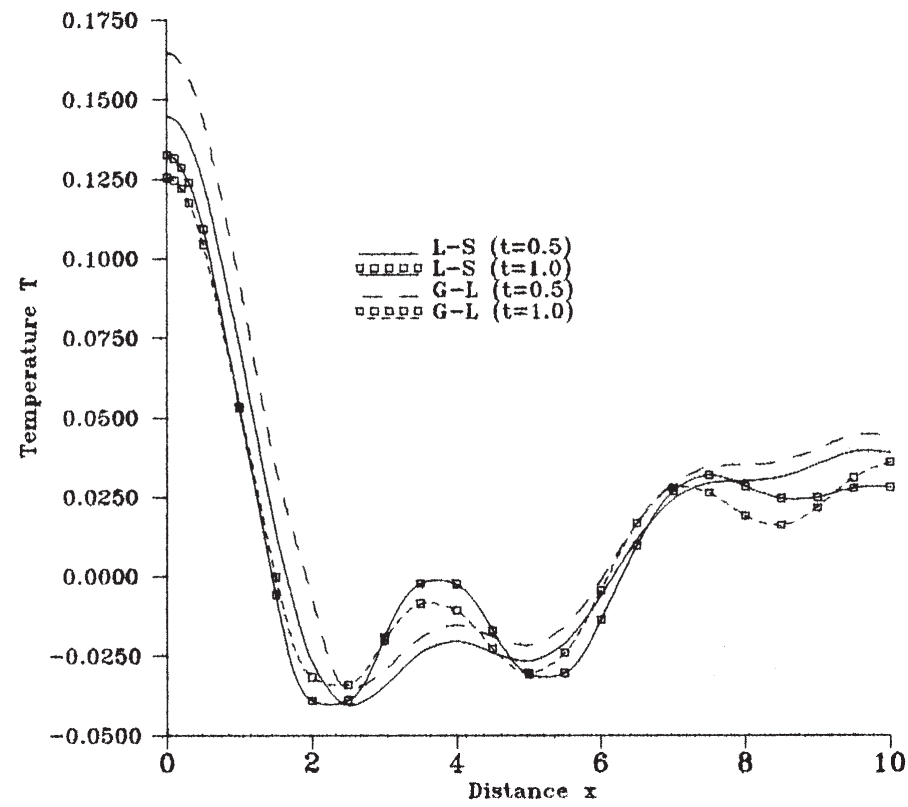

Figure 10. Variation of temperature $T$ with distance $x$ due to uniformly distributed thermal source (temperature gradient boundary). 


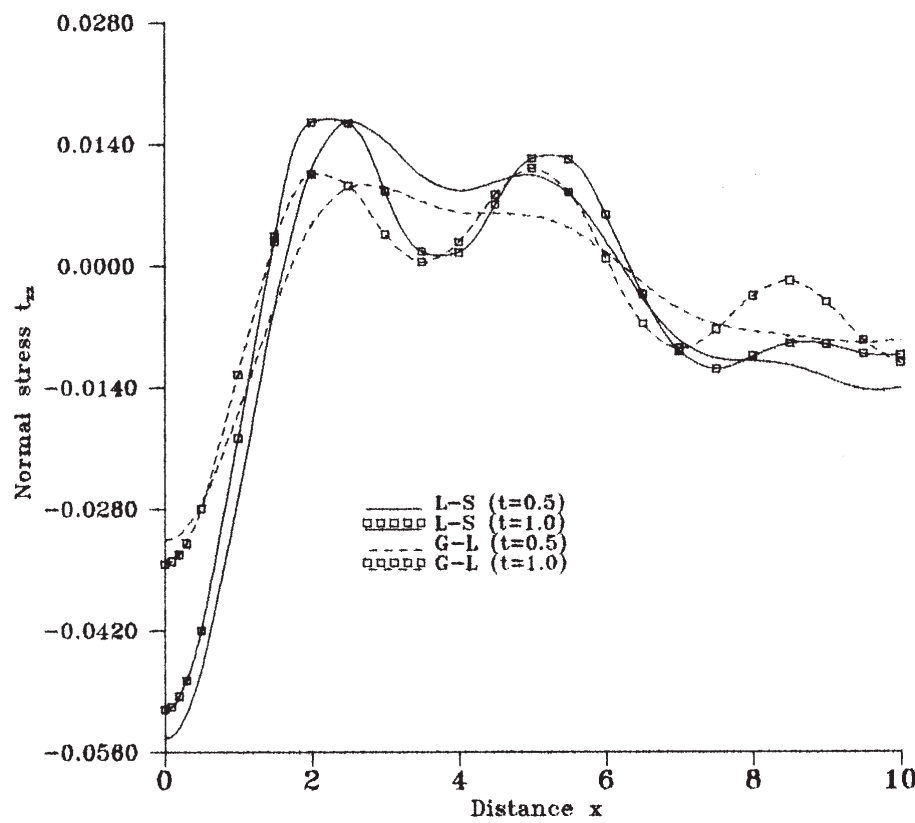

Figure 11. Variation of normal stress $t_{z z}$ with distance $x$ due to linearly distributed thermal source (temperature gradient boundary).

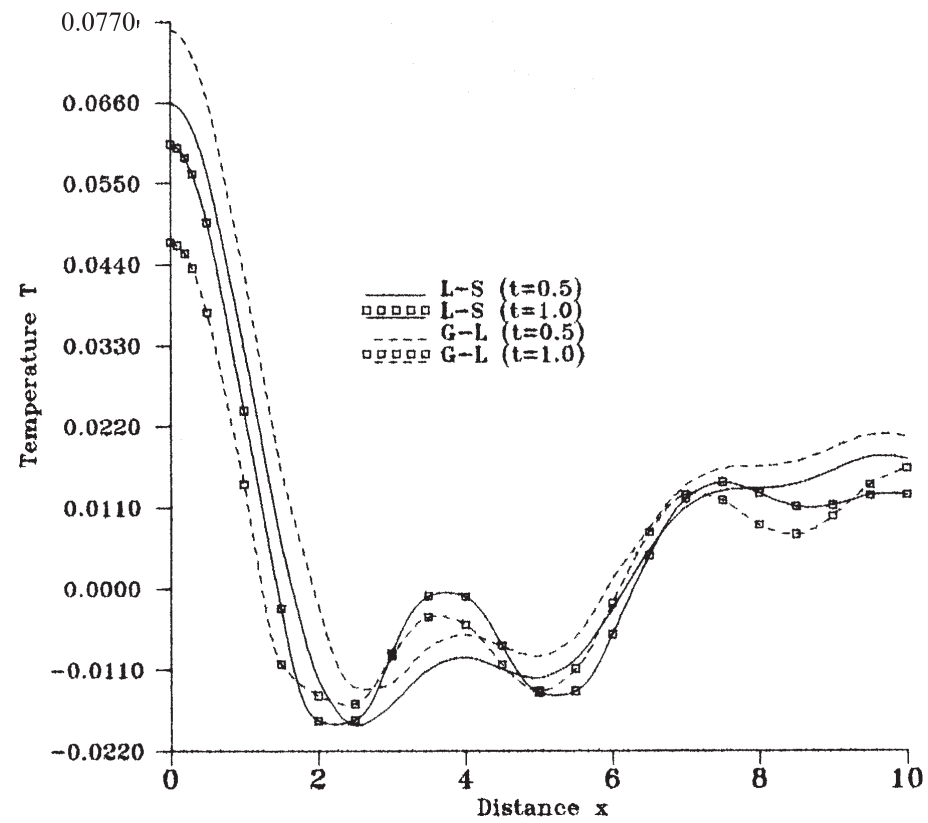

Figure 12. Variation of temperature $T$ with distance $x$ due to linearly distributed thermal source (temperature gradient boundary). 


\section{Conclusions}

(1) The Laplace and Fourier transform technique is used to derive displacements, stresses and temperature distribution due to mechanical and thermal sources.

(2) The comparison of different theories of thermoelasticity, i.e. L-S (Lord \& Shulman 1967) and G-L (Green \& Lindsay 1972) theories for insulated and temperature gradient boundary is carried out.

(3) As $x$ diverges from the point of application of source the components of normal stress and temperature distribution are observed to follow oscillatory patterns.

(4) Normal stress and temperature distribution show opposite oscillatory behaviour for concentrated, uniformly and linearly distributed forces.

(5) It is observed that magnitudes of normal stress and temperature distribution are large near the point of application of source. It is also observed that for insulated boundary and temperature gradient boundary, the behaviour variations of normal stress and temperature distribution for concentrated force (mechanical) and thermal point source is similar to that of distributed load (mechanical) and distributed thermal of source with only differences in their magnitude.

\section{List of symbols}

$\begin{array}{ll}c_{i j k l} & \text { isothermal elastic parameters; } \\ e_{i j} & \text { strain tensor; } \\ T_{0} & \text { uniform temperature, } \\ T(x, y, z, t) & \text { temperature change; } \\ t & \text { time; } \\ t_{i j} & \text { stress tensor; } \\ \overrightarrow{\mathbf{u}}=(u, v, w) & \text { displacement vector; } \\ \alpha_{k l} & \text { linear thermal expansion tensor; } \\ \delta_{i j} & \text { Kronecker's delta; } \\ \tau_{0}, \tau_{1} & \text { thermal relaxation times; } \\ \rho & \text { density. }\end{array}$

\section{References}

Banerjee D K, Pao Y H 1974 Thermoelastic waves in anisotropic solids. J. Acoust. Soc. Am. 56: 1444-1453

Barnett D M , Lothe J 1974 Consideration of the existence of surface wave (Rayleigh wave) solutions in anisotropic elastic crystals. J. Phys. F4: 671-686

Chadwick P 1976 The existence of pure surface modes in elastic materials with orthorhombic symmetry. J. Sound Vibr. 47: 39-52

Destrade M 2001 The explicit secular equation for surface acoustic waves in monoclinic elastic crystals. J. Acoust. Soc. Am. 109: 1398-1402

Dhaliwal R S, Sherief H H 1980 Generalised thermoelasticity for anisotropic media. Q. Appl. Math. 38: $1-8$

Dhaliwal R S, Singh A 1980 Dynamic coupled thermoelasticity (New Delhi: Hindustan) p. 726

Domanski W, Jablonski T 2001 On resonances of nonlinear elastic waves in cubic crystal. Arch. Mech. 53: $91-104$ 
Green A E, Lindsay K A 1972 Thermoelasticity. J. Elasticity 2: 1-7

Honig G, Hirdes U 1984 A method for the numerical inversion of Laplace transform. J. Comput. Appl. Math.10: 113-132

Lin W, Zhao Y-Q 1995 On the plane problem of orthotropic quasi-static thermoelasticity. J. Elasticity 41: 161-175

Lord H W, Shulman Y 1967 A generalised dynamical theory of thermoelasticity. J. Mech. Phys. Solids 15: 299-309

Musgrave M J P 1981 On an elastodynamic classification of orthorhombic media. Proc. R. Soc. Lond. A. 374: 401-429

Pao Y H, Banerjee D K 1973 Thermal pulses in dielectric crystals. Lett. Appl. Eng. Sci. 1: 33-41

Press W H, Teukolshy S A, Vellerling W T, Flannery B P 1986 Numerical recipes (Cambridge: University Press)

Royer D, Dieulesaint E 1984 Rayleigh wave velocity and displacement in orthorhombic, tetragonal, hexagonal and cubic crystals. J.Acoust. Soc. Am. 76: 1438-1444

Singh H, Sharma J N 1984 Generalised thermoelastic waves in transversely isotropic media. J.Acoust. Soc. Am. 77: 1046-1053

Sharma J N, Singh H 1990 Propagation of generalised thermoelastic waves in cubic crystals. Arch. Mech. 42: 19-30

Sharma J N, Kumar V, Sud S P 2000 Plane harmonic waves in orthorhombic thermoelastic material. J. Acoust. Soc. Am. 107: 293-305

Verma K L, Hasebe N 2001 Wave propagation in plates of general anisotropic media in generalised thermoelasticity. Int. J. Eng. Sci. 39: 1739-1763 